\title{
NF2 deficiency promotes tumorigenesis and metastasis by destabilizing adherens junctions
}

\author{
Dominique Lallemand, ${ }^{1}$ Marcello Curto, ${ }^{1}$ Ichiko Saotome, ${ }^{1}$ Marco Giovannini, ${ }^{2}$ \\ and Andrea I. McClatchey ${ }^{1,3}$ \\ ${ }^{1}$ MGH Cancer Center and Harvard Medical School Department of Pathology, Charlestown, Massachusetts 02129, USA; \\ ${ }^{2}$ INSERM 434, Fondation Jean Dausset-CEPH, 75010, Paris, France
}

\begin{abstract}
Mutation of the Neurofibromatosis 2 (NF2) tumor suppressor gene leads to cancer development in humans and mice. Recent studies suggest that $N f 2$ loss also contributes to tumor metastasis. The Nf2-encoded protein, merlin, is related to the ERM (ezrin, radixin, and moesin) family of membrane:cytoskeleton-associated proteins. However, the cellular mechanism whereby merlin controls cell proliferation from this location is not known. Here we show that the major cellular consequence of $\mathrm{Nf2}$ deficiency in primary cells is an inability to undergo contact-dependent growth arrest and to form stable cadherin-containing cell:cell junctions. Merlin colocalizes and interacts with adherens junction (AJ) components in confluent wild-type cells, suggesting that the lack of AJs and contact-dependent growth arrest in $\mathrm{Nf}^{-/-}$cells directly results from the absence of merlin at sites of cell:cell contact. Our studies indicate that merlin functions as a tumor and metastasis suppressor by controlling cadherin-mediated cell:cell contact.
\end{abstract}

[Keywords: NF2; merlin; tumor suppressor; metastasis; cytoskeleton; adherens junction]

Received October 29, 2002; accepted in revised form March 6, 2003.

Neurofibromatosis type 2 (NF2) is a familial cancer syndrome that features the development of multiple nervous system tumors including schwannomas and meningiomas (Gutmann 1997). The disease is caused by inherited heterozygous mutation of the NF2 tumor suppressor gene; somatic loss of the wild-type allele leads to tumor development. The study of Nf2-mutant mice shows that the NF2 tumor suppressor regulates the proliferation of many cell types and that Nf2 loss plays a broad role in both tumor development and in tumor metastasis (McClatchey et al. 1997, 1998; Giovannini et al. 2000; Kalamarides et al. 2002). Despite the cloning of the NF2 gene a decade ago, the molecular function of its encoded protein, merlin, is not yet known. Merlin is a member of the ERM (ezzrin, radixin, and moesin) family of proteins that link the actin cytoskeleton to various membrane-associated proteins (Bretscher et al. 2002; Sun et al. 2002). Like the ERM proteins, merlin localizes to the membrane:cytoskeleton interface and thus occupies an unusual physical location for a tumor suppressor. Merlin has been reported to interact with a number of protein partners and to inhibit many signaling pathways when overexpressed (Bretscher et al. 2002; Sun et al.

${ }^{3}$ Corresponding author.

E-MAIL mcclatch@helix.mgh.harvard.edu; FAX (617) 726-7808.

Article published online ahead of print. Article and publication date are at http://www.genesdev.org/cgi/doi/10.1101/gad.1054603.
2002). However, it is not clear which, if any, of the properties of overexpressed merlin are relevant to the tumorigenic consequences of its loss of function.

Somatic loss of NF2 function is causal to tumor development in mice and humans. Therefore, important clues to the molecular function of merlin can be derived from an analysis of the cellular and molecular consequences of NF2 deficiency. Although some properties of human NF2-deficient tumor cells have been reported, genetically matched control cells are not readily available for comparison (Pelton et al. 1998). The consequences of Nf2 deficiency in primary cells have not been examined. We previously generated Nf2-mutant mice, which are a valuable source of primary Nf2-deficient and control cells (McClatchey et al. 1997; Giovannini et al. 2000). In this study, we have carefully examined the properties of primary Nf2-deficient mouse embryo fibroblasts (MEFs) and found that they are not transformed and, in fact, are indistinguishable from wild-type MEFs in many ways. However, unlike wild-type MEFs, Nf2-deficient MEFs do not undergo contact-dependent inhibition of growth. We found that in both MEFs and in primary epithelial keratinocytes, Nf2 deficiency leads to defective cadherin-mediated cell:cell adhesion. Merlin localizes to adherens junctions (AJs) and physically interacts with AJ components in confluent wild-type cells, suggesting that merlin normally controls AJ assembly and contact-dependent growth inhibition directly from sites of cell:cell 
contact. These results suggest that loss of $\mathrm{AJ}$ function is the cellular mechanism whereby $N f 2$ deficiency leads to tumor and metastasis development.

\section{Results}

Heterozygous Nf2-mutant mice develop a variety of tumor types, including fibrosarcomas; therefore, we initially studied the growth properties of Nf2-deficient primary mouse embryo fibroblasts. We previously determined that both the levels and phosphorylation of merlin are regulated by three conditions in cultured immortalized and primary fibroblasts: high cell density, growth factor withdrawal, and cell:ECM detachment (Shaw et al. 1998; data not shown). Therefore, we asked whether merlin is required for normal growth arrest under these culture conditions. Subconfluent, exponentially growing wild-type and $\mathrm{N}^{-2^{-/}} \mathrm{MEF}$ exhibited identical rates of growth and comparable levels of S-phase entry, suggesting that Nf2 deficiency does not cause an increase in the rate of proliferation of these cells per se (Fig. 1A). However, although wild-type MEFs stopped dividing after reaching confluence, $\mathrm{Nf2^{-/- }}$ MEFs continued to proliferate without saturating (Fig. 1A). In contrast to the monolayer formed by confluent wild-type cells, $N f 2^{-/-}$cells formed a multilayer that eventually detached from the culture dish, preventing the measure of later time points. Flow cytometry confirmed that in contrast to wild-type MEFs, a significant percentage of $N f 2^{-/-}$MEFs continued to enter S phase after reaching confluence (Fig. 1B). Thus, $\mathrm{Nf}^{-/-} \mathrm{MEFs}$ do not undergo contact-dependent inhibition of proliferation.
Further study revealed that Nf2-deficient MEFs are not generally defective in their ability to arrest growth. Both wild-type and $\mathrm{Nf2^{-/ }}$ cultures underwent complete growth arrest after $8 \mathrm{~h}$ in suspension or $120 \mathrm{~J}$ of UVC irradiation (Fig. 1B). In fact, although a role for merlin function in cell:ECM adhesion has been proposed (Obremski et al. 1998; Shaw et al. 1998; Gutmann et al. 1999; Fernandez-Valle et al. 2002; Johnson et al. 2002), we could not detect obvious cell:ECM adhesion defects in $\mathrm{Nf2} 2^{-/-}$MEFs. Thus, both wild-type and $\mathrm{Nf2}^{-/-}$MEFs attach to and spread on various ECM substrates with comparable kinetics; similarly, focal adhesion formation and density are grossly normal in $\mathrm{Nf2} 2^{-/-}$MEFs (data not shown). This is consistent with our observation that $\mathrm{Nf2}^{-{ }^{-}}$MEFs are not transformed and cannot form colonies in soft agar or tumors in nude mice (I. Saotome and A. McClatchey, unpubl.). Interestingly, $N f 2^{-/-} \mathrm{MEFs}$ also did not undergo complete growth arrest when serumstarved. Instead, they continued to proliferate slowly, surviving for 2-3 wk without added growth factors (data not shown). Together these data suggest that merlin normally mediates growth arrest specifically under conditions of growth-factor deprivation and cell:cell contact.

The primary phenotype exhibited by $\mathrm{Nf}^{-/-}$MEFs was a failure to undergo contact-dependent growth arrest. Examination of key mitogenic signaling pathways in wildtype and $\mathrm{Nf}^{-/-} \mathrm{MEF}$ at low and high cell density provided a molecular correlation of this growth advantage. As expected given their similar rates of growth, equivalent levels and/or phosphorylation of many signaling proteins were detected in exponentially growing wildtype and $\mathrm{Nf2}^{-/-}$MEFs (data not shown). In contrast,

$\mathbf{A}$

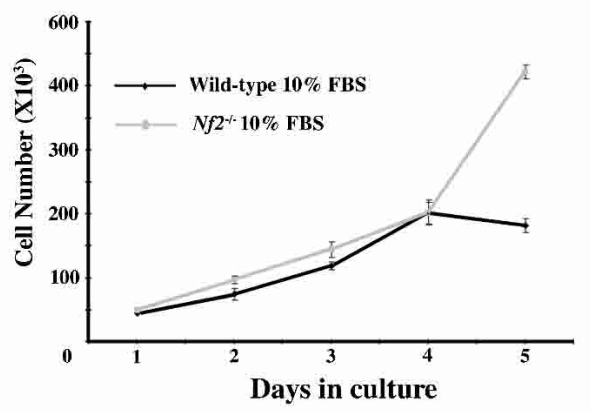

B

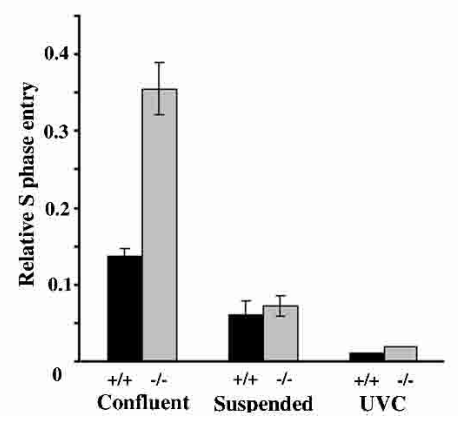

C

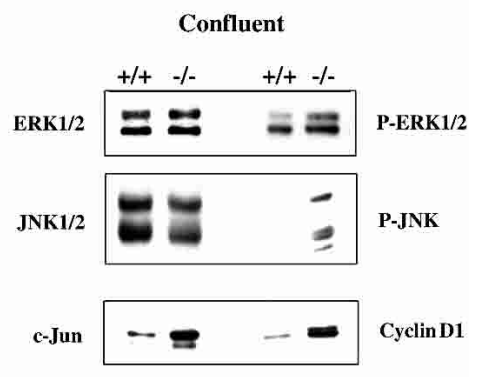

Figure 1. $N f 2^{-/-}$MEFs do not undergo contact-dependent inhibition of proliferation. $(A)$ Wild-type and $\mathrm{Nf}^{-/-}$MEFs cultured in the presence of growth factors were counted daily for $5 \mathrm{~d}$. (B) Growth arrest of $\mathrm{Nf2^{-/- }}$ MEFs by high density but not loss of adhesion or UVCirradiation is impaired. Subconfluent, confluent, suspended, or UVC-treated wild-type and $\mathrm{Nf}^{-/-} \mathrm{MEF}$ were labeled with BrdU and analyzed by fluorescent-activated cell sorting (FACs) analysis. The graph displays the relative reduction in $S$ phase of wild-type and $\mathrm{Nf}^{-/-}$MEFs compared with subconfluent MEFs of the same genotype. Equivalent percentages of wild-type and $\mathrm{Nf}^{-/-}$cells remained viable in these experiments (data not shown). FACs analysis also revealed that wild-type and $\mathrm{Nf}^{-{ }^{--}}$MEFs have equivalent cell volumes (data not shown). (C) The level and phosphorylation of key mitogenic signaling molecules were examined in confluent (unstarved) wild-type and $\mathrm{Nf2^{-/- }}$ MEFs by Western blot. Elevated levels of cyclin D, cjun, and phosphorylated (active) ERK1/2, JNK persist in confluent $\mathrm{Nf2}^{-/-} \mathrm{MEFs}$. 
whereas the levels and/or phosphorylation of key signaling proteins markedly diminished after wild-type cells reached confluence, they remained elevated in confluent $N f 2^{-/-}$cells, consistent with their persistent proliferation. For example, the levels of cyclin D1, c-jun, phosphorylated ERK1/2, and phosphorylated JNK remained elevated in $N f 2^{-/-}$MEFs after reaching confluence (Fig. 1C). Thus, Nf2-deficient cells fail to down-modulate mitogenic signaling and continue to proliferate at high cell density.

The ability of confluent $N f 2^{-/-}$MEFs to pile up on top of one another, apparently failing to recognize their immediate neighbors, is apparent upon visual inspection (Fig. 2A). Several studies detail a direct role for AJs in contact-dependent inhibition of epithelial cell proliferation (for reviews, see Nollet et al. 2000; Cavallaro and Christofori 2001). Cadherin family adhesion proteins mediate homotypic interaction between adjacent cells and are central to the assembly and function of AJs. Cadherins cluster and recruit intracellular proteins such as catenins $(\alpha$-catenin, $\beta$-catenin, and p120) and the actin cytoskeleton to AJs (for reviews, see Nagafuchi 2001; Perez-Moreno et al. 2003). AJs have also been identified and studied in fibroblasts by both immunologic and electron microscopic criteria (Yonemura et al. 1995; Gloushankova et al. 1998). To determine whether merlin is involved in the establishment or maintenance of these structures, we examined the expression of AJ components and the assembly of AJs in confluent, wild-type, and $N f 2^{-/-}$MEFs. Indeed, both wild-type and $\mathrm{Nf2} 2^{-/-}$ MEFs express equivalent levels of the core AJ components $\mathrm{N}$-cadherin, $\alpha$-catenin, and $\beta$-catenin (Fig. 2B). Low levels of E-cadherin were also detected in both wild-type, and $\mathrm{Nf2}^{-{ }^{-}}$MEFs (data not shown). In wild-type fibroblasts, indirect immunofluorescence revealed the punctate localization of $\beta$-catenin along boundaries of cellcell contact as has been described (Fig. 2C; Yonemura et al. 1995; Gloushankova et al. 1998). Other AJ components, including N-cadherin, exhibited identical staining (data not shown). In contrast, we never observed AJ-like structures in $\mathrm{Nf2}^{-/-} \mathrm{MEFs}$; instead, all AJ components examined were diffusely localized throughout the membrane despite extensive areas of contact between cells (Fig. 2C, right; data not shown).

To establish that the loss of contact-dependent inhibition of proliferation and loss of AJs were a direct result of the loss of merlin function, we examined the consequences of merlin reintroduction into these cells. We previously found that phosphorylation of C-terminal residue S518 leads to relocalization and inactivation of merlin, whereas an unphosphorylatable mutant (S518A) version functions as a constitutively active protein (Shaw et al. 2001). In MEFs, both endogenous and exogenous merlin are predominantly hyperphosphorylated, suggesting that the availability of active, hypophosphorylated merlin is tightly controlled (data not shown). To ensure that reintroduction of active merlin was achieved, we infected $N f 2^{-/-}$MEFs with an adenovirus expressing $N f 2^{S 518 A}$ (Ad-Nf2 ${ }^{S 518 A}$ ). As shown in Figure $2 \mathrm{C}, \mathrm{Nf} 2^{S 518 A}$ expression restored both contact-depen-
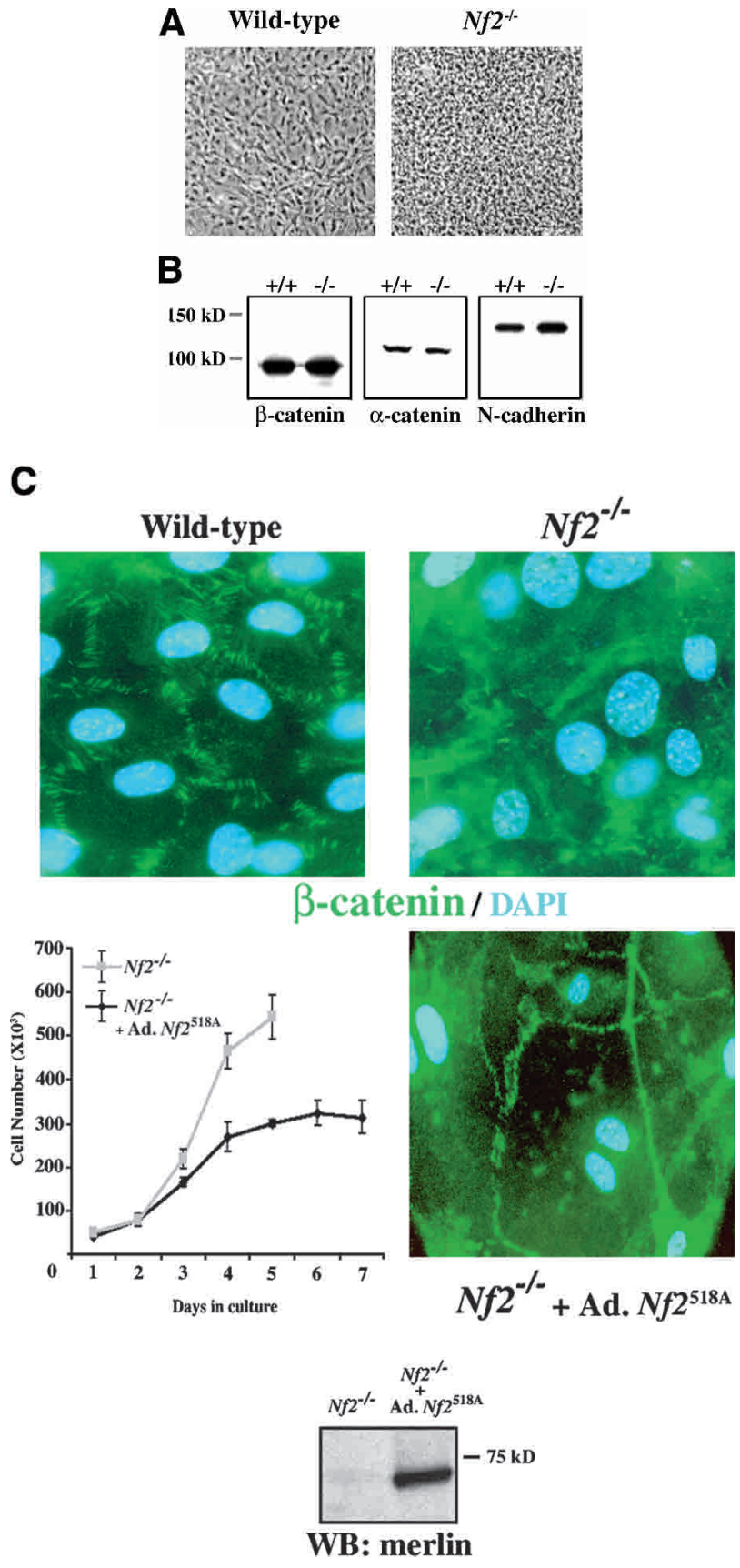

Figure 2. Loss of AJs in $\mathrm{Nf2}^{-/-}$MEFs. (A) Wild-type and $\mathrm{Nf2^{-/- }}$ MEFs were cultured in the presence of growth factors for $4 \mathrm{~d}$ after reaching confluence and photographed by phase contrast. $(B)$ Expression of $\beta$-catenin, $\alpha$-catenin, and $\mathrm{N}$-cadherin in confluent wild-type and $\mathrm{Nf2}^{-/-} \mathrm{MEFs}$ was analyzed by Western blot analysis. (C) Wild-type and $\mathrm{Nf}^{-/-}$MEFs were grown to confluence and starved for $48 \mathrm{~h}$. Immunofluorescent localization of $\beta$-catenin revealed punctate staining along cell:cell boundaries in wild-type but not $\mathrm{Nf}^{-1-}$ MEFs. The lower panels show the rescue of both contact-dependent inhibition of proliferation (left) and AJ formation (right) by reintroduction of active merlin $\left(\mathrm{Nf} 2^{\mathrm{S} 518 \mathrm{~A}}\right)$. Expression of $\mathrm{Nf2} 2^{\mathrm{S} 518 \mathrm{~A}}$ detected by Western blot is shown below. 
dent inhibition of proliferation and $\mathrm{AJ}$ formation to Nf2-/- MEFs.

To further test the dependency of $\mathrm{AJ}$ formation on merlin function we expressed a dominant-negative form of merlin known as the "Blue Box" mutant (Nf2 ${ }^{\Delta \mathrm{BB}}$ ) in wild-type MEFs (LaJeunesse et al. 1996). We found that confluent $N f 2^{\triangle B B}$-expressing MEFs piled up on top of one another and no longer exhibited punctate localization of AJ components at cell:cell boundaries (Fig. 3A). As predicted of a true dominant negative, $N f 2^{\Delta B B}$ expression had no detectable effect on the phenotype of $\mathrm{Nf2} 2^{-/-}$MEFs (data not shown). Thus, expression of a dominant-negative version of merlin mimics $N f 2$ deficiency and interferes with the ability of wild-type cells to assemble AJs, confirming the specificity of merlin function in this process.

It has recently been reported that merlin controls contact inhibition of rat schwannoma cells through interaction with CD44 (Morrison et al. 2001). To explore the relationship between merlin and CD44 in MEFs, we measured the ability of $c d 44^{-/-}$MEFs to undergo contact-dependent inhibition of proliferation. In contrast to $\mathrm{Nf2}^{-/-}$MEFs, $\mathrm{cd} 44^{-/-}$MEFs stopped proliferating upon reaching confluence with a saturation density similar to that of wild-type MEFs (Fig. 3B). Moreover, $\beta$-catenin localization to cell:cell boundaries in $c d 44^{-/-}$MEFs was indistinguishable from wild type (Fig. 3A). Therefore, the loss of contact-dependent growth arrest in $\mathrm{Nf2}^{-/-}$MEFs appears to be CD44-independent.

Finally, to verify that the loss of contact-dependent growth arrest and absence of AJs in $\mathrm{Nf2}^{-/-}$cells is not simply an indirect consequence of increased mitogenic signaling, we examined MEFs lacking the NF1 tumor suppressor, which is a negative regulator of ras signaling (Cichowski and Jacks 2001). As in other cell types, Nf1 deficiency in MEFs leads to a proliferative advantage that is likely caused by increased ras signaling (Cichowski et al. 2003; D. Lallemand and A. McClatchey, unpubl.). However, $\mathrm{Nf1}^{-/-} \mathrm{MEFs}$ do undergo contact-dependent inhibition of growth and form numerous AJs along cell:cell boundaries (Fig. 3A,B). This is consistent with the observation that ras-transformed Rat-1 fibroblasts also form AJs (Gloushankova et al. 1998). Thus, the lack of AJ formation is specific to loss of merlin function.

Most reports of merlin localization to areas of cortical
A
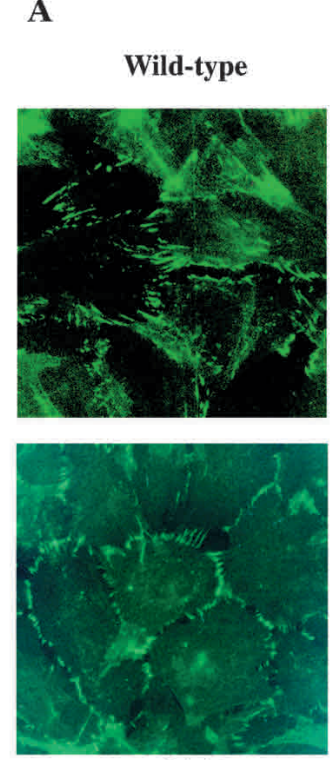

$c d 44^{\%}$

B

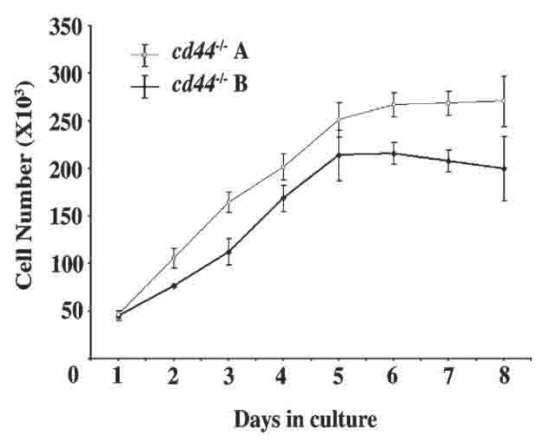

Wild-type Ad. $\stackrel{+}{N} f 2^{\text {ABB }}$
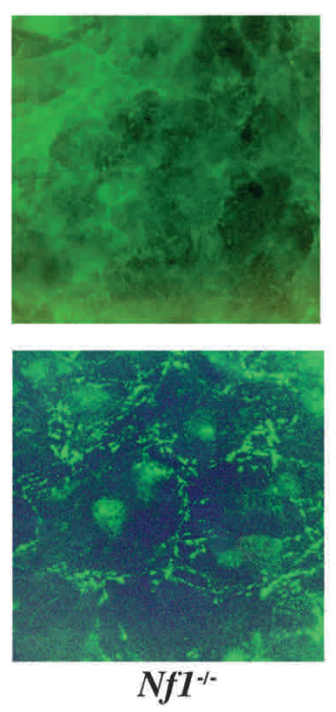

Nf2:

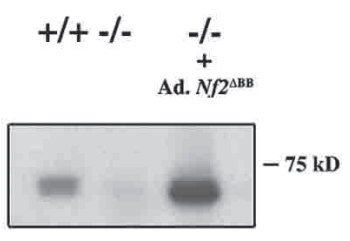

WB: merlin
Figure 3. Expression of dominant-negative merlin $\left(N f 2^{\triangle B B}\right)$ disrupts $\beta$-catenin localization in wild-type MEFs. (A) Immunofluorescence staining of $\beta$-catenin in confluent, serum-starved $c d 44^{-/-}$MEFs, $\mathrm{Nf1^{-/ }}$ MEFs, or wild-type MEFs infected with an adenovirus expressing $N f 2^{\triangle B B}$. Expression of dominant-negative merlin disrupts $\beta$-catenin localization in wild-type cells (top right). In contrast, $\beta$-catenin localizes normally in $c d 44^{-/-}$(bottom left) or $N f 1^{-1-}$ (bottom right) MEFs. Expression of $\mathrm{Nf} 2^{\triangle B B}$ in $\mathrm{Nf2} 2^{-/-}$MEFs was demonstrated by Western blot using an anti-merlin antibody (right). (B) $\mathrm{Cd}_{4} 4^{-1-}$ and $\mathrm{Nf1^{-/- }} \mathrm{MEFs}$ exhibit contact-dependent inhibition of growth. $c d 44^{-/-}$(two independently prepared populations, $\mathrm{A}$ and $\mathrm{B}$ ) and $\mathrm{Nf1}^{-/-}$ MEFs were seeded at a subconfluent density in the presence of growth factors and counted daily. After 4-5 d, MEFs of both genotypes reached saturation and stopped proliferating. 
cytoskeletal remodeling such as membrane ruffles describe studies performed in subconfluent cells (Sun et al. 2002); however, merlin also localizes to areas of cell:cell contact in vivo in Drosophila and in cultured mammalian cells (McCartney and Fehon 1996; Maeda et al. 1999). To determine whether the lack of AJs in $\mathrm{Nf2}^{-/-}$ MEFs is a direct consequence of the absence of merlin from that location, we examined the localization of merlin in confluent wild-type MEFs by immunofluorescence. We detected precise colocalization of endogenous merlin with several AJ components, including $\beta$-catenin, $\mathrm{N}$-cadherin, and $\alpha$-catenin in punctate structures along borders of cell:cell contact (Fig. 4A; data not shown).

The possibility that merlin physically associates with AJ components was tested by immunoprecipitating merlin from confluent wild-type MEFs using two different anti-merlin antibodies; subsequent Western blotting revealed coimmunoprecipitation of $\beta$-catenin with merlin using either antibody (Fig. 4B, lanes 2,3). It is notable that these proteins were coimmunoprecipitated from the soluble membrane fraction, which contains only a small pool of merlin (Fig. 5A). We were unable to develop satisfactory conditions for immunoprecipitating merlin from the Triton-insoluble membrane fraction. In fact, we would predict that active hypophosphorylated merlin, which is underrepresented in the soluble fraction, preferentially interacts with $\beta$-catenin. Thus the data shown in Figure 4B may substantially underrepresent the magnitude of association between these two proteins. Notably, although merlin has been reported to interact with paxillin (Fernandez-Valle et al. 2002), we could not detect colocalization, cofractionation, or coimmunoprecipitation of merlin with paxillin in MEFs (Fig. 5A; data not shown). Together these data indicate that merlin associates with $\beta$-catenin at sites of cell:cell contact; loss of merlin from this location may directly impair the formation or stability of AJs, leading to loss of contactdependent inhibition of growth.

To explore the mechanistic relationship between merlin and AJs, we examined the subcellular distribution of AJ components in $\mathrm{Nf}^{-/-}$MEFs. We found that the distribution of AJ components among cytosolic, soluble membrane and insoluble membrane compartments was unaltered in $\mathrm{Nf2}^{-/-}$MEFs (Fig. 5A). In fact, core AJ complexes can be immunoprecipitated from the membrane of $\mathrm{Nf}^{-{ }^{--}}$cells at wild-type levels and stoichiometry (Fig. 5B). However, under conditions in which the cytosolic compartment has been depleted and membranes have been preserved, immunofluorescent localization of these components reveals diffuse distribution throughout the membrane of $\mathrm{Nf2}^{-/-}$cells (Figs. 2C, 3A). Therefore, loss of merlin does not prevent membrane localization or retention of the AJ core complex; instead, merlin appears to be required to assemble or stabilize the final AJ structure.
Figure 4. Merlin and $\beta$-catenin colocalize and associate with $\mathrm{AJ}$ components. (A) Localization of merlin (red) in confluent wild-type MEFs along a boundary of cell: cell contact (arrows; 20× magnification). Nuclei are stained with DAPI (blue). (B) Colocalization of merlin (red, arrows in left panel) and $\beta$-catenin (green, middle) in confluent wild-type MEFs at sites of cell:cell contact (merge, right panel; 40× magnification). This staining is not detectable in $\mathrm{Nf2}^{-/-}$MEFs (data not shown). (C) Immunoprecipitation of $\beta$-catenin (lane 1) and merlin $(\mathrm{N}$ terminal epitope, lanes 2,4; C-terminal epitope, lanes 3,5) from the membrane soluble fraction of wild-type (left panel) and $\mathrm{Nf2}^{-/-}$(right panel) MEFs, followed by Western blot (WB) analysis using anti- $\beta$-catenin (upper panel I) or anti-merlin (lower panel II) antibodies. (Lane 6) Total membrane soluble fraction from $\mathrm{Nf2}^{-/-} \mathrm{MEFs}$. In wild-type MEF membranes, $\beta$-catenin coimmunoprecipitates with merlin using both anti-merlin antibodies. As expected, $\beta$-catenin was not detected when immunoprecipitations from $\mathrm{Nf}^{-/-}$membranes were performed using anti-merlin antibodies (lanes 4,5).
A

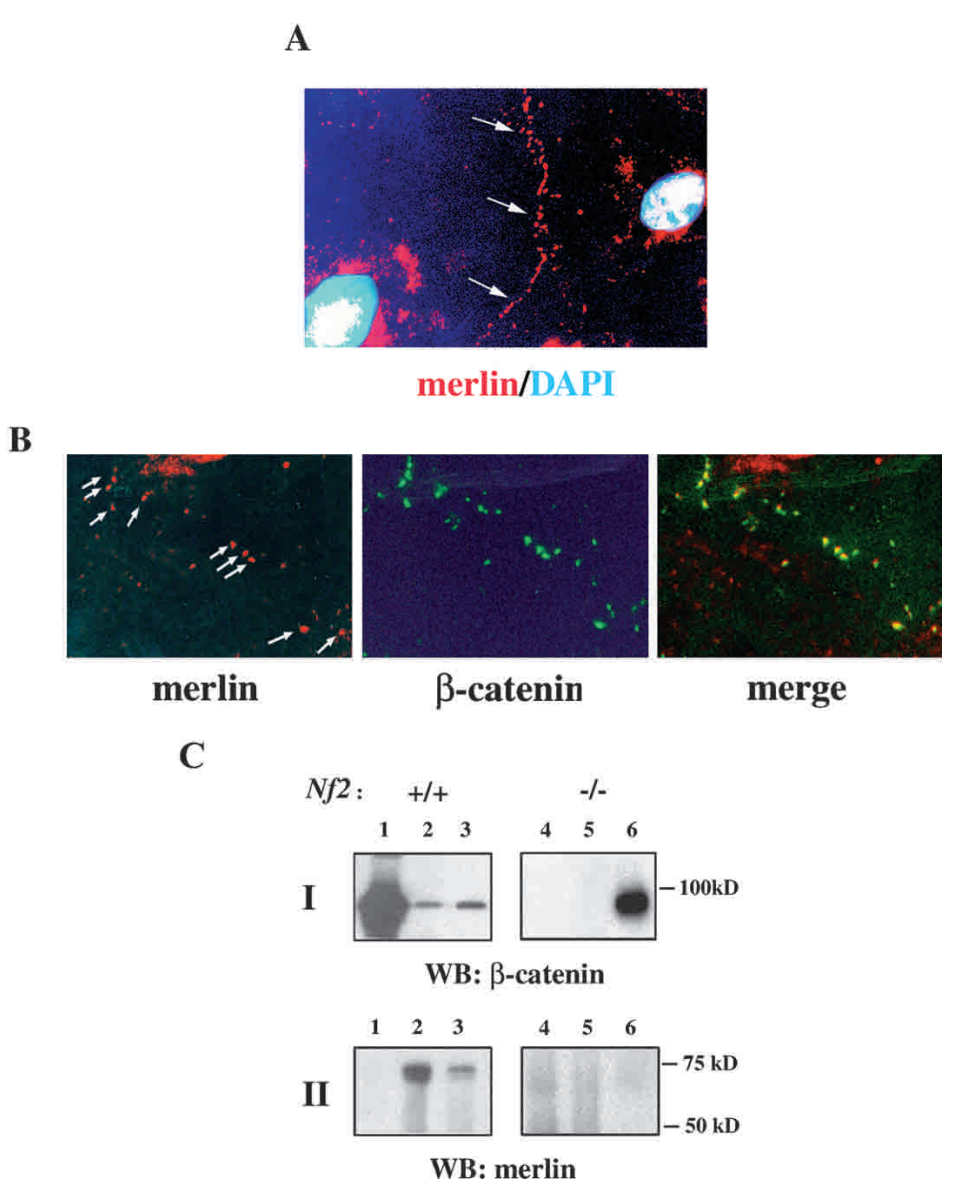

B 
A

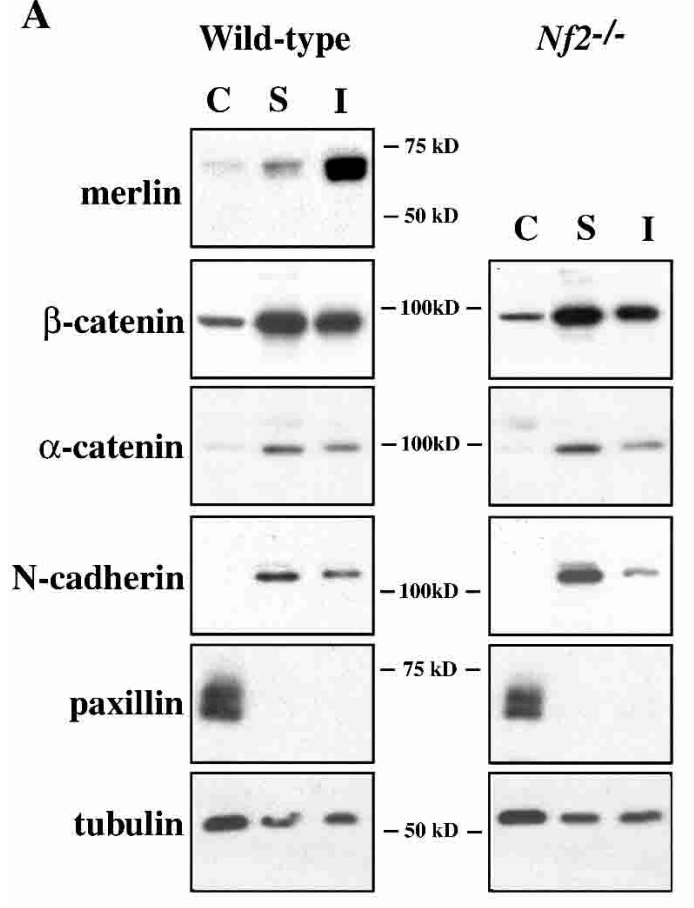

B

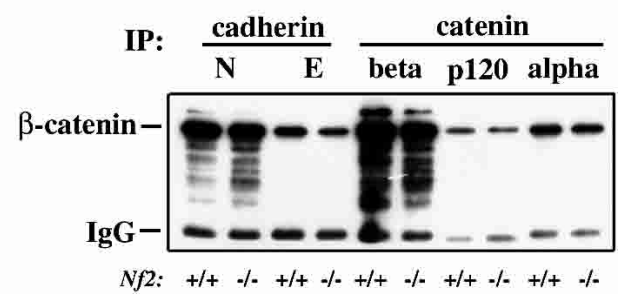

Figure 5. Core AJ complexes form in $\mathrm{Nf2}^{-/-} \mathrm{MEF}$ membranes. (A) Merlin and AJ component distribution in cytosolic (C), membrane soluble (S), and membrane insoluble (I) fractions from confluent, serum-starved wild-type (left) and $\mathrm{Nf2}^{-/-}$(right) MEFs. The distribution of AJ components is identical in wildtype and $\mathrm{Nf}^{-/-}$MEFs. Merlin is largely insoluble and cofractionates with $\beta$-catenin but not paxillin. (B) Equivalent levels of $\beta$-catenin coimmunoprecipitate with $\mathrm{N}$-cadherin, E-cadherin, $\beta$-catenin, p120 catenin, or $\alpha$-catenin from confluent wild-type and $N f 2^{-/-}$membranes.

Although their existence has been well documented in fibroblasts, AJ formation has been more thoroughly studied in epithelial cells. We therefore asked whether merlin is also required for AJ formation in primary epithelial mouse keratinocytes in which AJ assembly and disassembly can be controlled by modulating extracellular calcium levels (Vasioukhin et al. 2000). As shown in Figure 6A, in high $\mathrm{Ca}^{2+}$-containing medium, endogenous merlin precisely colocalizes with AJ components along cell:cell boundaries in these cells. To examine the localization of merlin in real time, we transfected wild-type primary keratinocyte cultures with a plasmid expressing a GFP-merlin fusion protein (GFP-Nf2 ${ }^{\mathrm{wt}}$ ). Like endogenous merlin, GFP-Nf2 ${ }^{\text {wt }}$ colocalizes with AJ compo- nents along developing and mature cell:cell boundaries (Fig. 6B).

To determine whether localization to AJs is critical for the tumor suppressor function of merlin, we expressed a version of merlin carrying a rare patient-derived missense mutation (GFP-Nf2 ${ }^{\mathrm{L} 64 \mathrm{P}}$ ) in primary keratinocytes. This mutation is known to abrogate the growth-suppressing function of merlin; however, the subcellular localization of $\mathrm{Nf} 2^{\mathrm{L} 64 \mathrm{P}}$ has not been evaluated (Gutmann et al. 1998; Morrison et al. 2001). In striking contrast to wild-type merlin, GFP-Nf2 ${ }^{\mathrm{L} 64 \mathrm{P}}$ exhibited a strong punctate distribution throughout the cytoplasm (Fig. 6C, right). Interestingly, at early time points after transfection (12 h), a small fraction of GFP-Nf2 ${ }^{\mathrm{L} 64 \mathrm{P}}$ did localize to cell:cell boundaries, suggesting that the mutation does not lead to gross mislocalization of merlin but, rather, prevents retention of merlin to AJs (Fig. 6C, left). Notably, AJs form normally in wild-type keratinocytes that produce GFP-Nf2 ${ }^{\text {L64P }}$ (data not shown); thus, GFP$\mathrm{Nf2}{ }^{\mathrm{L} 64 \mathrm{P}}$ does not function as a dominant-negative version of merlin. Together, these data suggest that stable localization to cell:cell boundaries is important for the growth-suppressing function of merlin.

Finally, we examined the formation of AJs in AdCre-infected primary keratinocytes from newborn $N f 2^{\text {flox2/flox2 }}$ mice. After Ad-Cre infection (3 d), cells were switched to high calcium to stimulate AJ formation. In wild-type cells at early time points after calcium induction (3-6 h), actin-containing zipper-like structures formed along cell:cell boundaries as has been described (Vasioukhin et al. 2000). These structures subsequently resolved into continuous zones of adhesion containing actin and AJ components (Fig. 6D). In contrast, Ad-Creinfected keratinocytes did not form adhesion zippers at early time points after calcium induction (data not shown). Instead, they became misshapen, losing the integrity of the cortical actin ring and exhibiting diffuse membrane localization of AJ components. Even $20 \mathrm{~h}$ after calcium induction, zones of adhesion were rarely, if ever, apparent in $\mathrm{Nf2}^{-/-}$keratinocytes, despite extensive physical contact between cells (Fig. 6D). Thus, as in fibroblasts, merlin function is required for the formation or stabilization of AJs in primary epithelial cells.

\section{Discussion}

Recent studies suggest that inactivation of the NF2 tumor suppressor merlin plays a broad role in cancer development and progression in humans and in mice (McClatchey et al. 1998). Insight into the basis of this awaits the definition of the molecular function of merlin. Here, we provide the first analysis of the phenotype associated with Nf2 loss of function in primary cells. We found that the primary phenotypic consequence of $N f 2$ deficiency is loss of contact-dependent inhibition of growth. Several lines of evidence suggest that this phenotype is due to a lack of AJ formation or maintenance. First, $N f 2^{-/-}$MEFs do not form AJs, despite normal expression and membrane localization of AJ components. Second, reintroduction of active merlin restores both contact-dependent in- 
Lallemand et al.

Figure 6. Merlin localization in primary keratinocytes. (A) Confluent wild-type keratinocytes were cultured in the presence of $2 \mathrm{mM} \mathrm{CaCl}_{2}$ for $20 \mathrm{~h}$ to stimulate the formation of AJs. Immunostaining revealed perfect colocalization of endogenous merlin (red) and $\beta$-catenin (green; merge, yellow). (B) A GFP-Nf2 (wildtype) fusion protein (green; middle) colocalizes with Ecadherin (red) to a region of nascent cell:cell contact in wild-type primary keratinocytes (merge, yellow). Transfection of the GFP-Nf2-expressing plasmid and calcium induction were carried out simultaneously; $24 \mathrm{~h}$ later, cells were fixed, permeabilized, and processed for immunofluorescence. $(C)$ A mutant GFP-Nf2 ${ }^{\mathrm{L} 64 \mathrm{P}}$ version of merlin exhibits limited localization to cell cell boundaries at early times (12 h) after transfection, but at late times $(48 \mathrm{~h})$ after transfection, GFP-Nf2 $2^{\mathrm{L} 64 \mathrm{P}}$ exhibits only punctate, cytoplasmic localization. $\mathrm{Nu}$ clei are stained with DAPI (blue). (D) In a wild-type keratinocyte monolayer (upper panels), $\beta$-catenin localizes precisely to cell:cell boundaries (right panel). In contrast, in $\mathrm{Nf}^{-/-}$keratinocytes (bottom panels), $\beta$-catenin is diffusely localized throughout the cell. In addition, rhodamine-phalloidin staining (left panels) reveals that the cortical actin ring is malformed and badly disorganized in $\mathrm{Nf2}^{-/-}$keratinocytes.

hibition of growth and AJ formation to $\mathrm{Nf2}^{-/-}$MEFs. Third, a dominant-negative version of merlin confers loss of AJ formation to wild-type MEFs. Fourth, merlin colocalizes and associates with AJ components. Finally, a patient-derived missense mutation abolishes both the
A

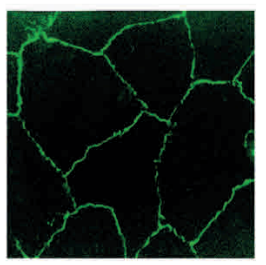

в

$\beta$-catenin

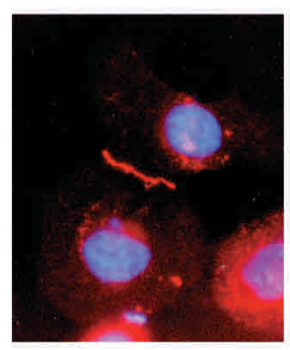

E-cadherin/DAPI

C

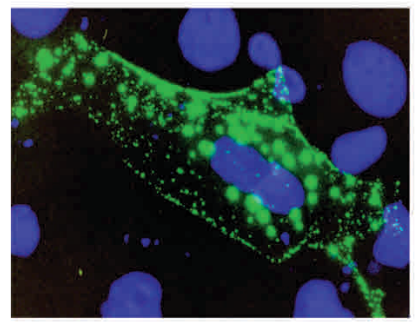

Early

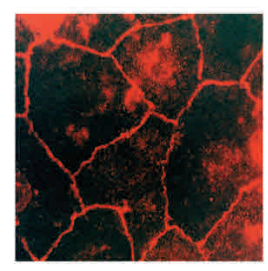

merlin

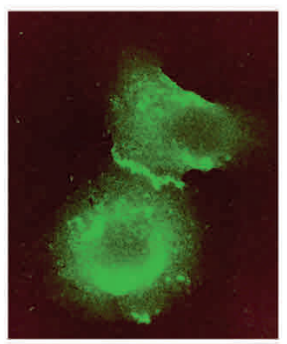

GFP-merlin

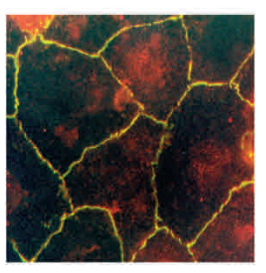

merge

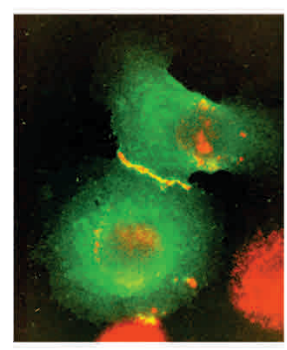

merge

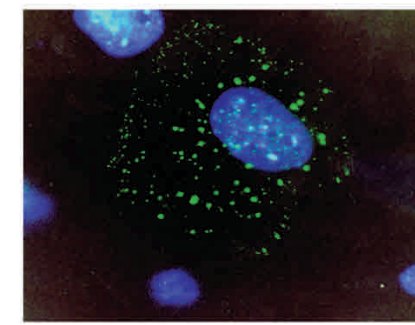

Late
GFP-merlin L64P/DAPI

D
Actin

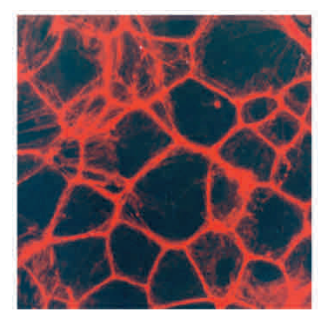

Wild-type

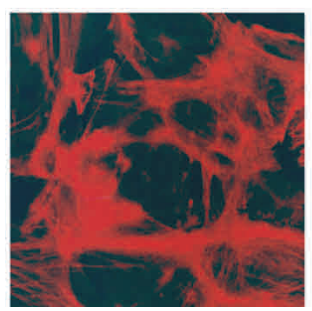

$\beta$-catenin
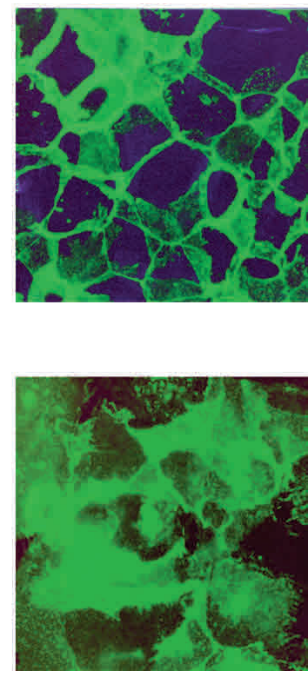

growth-suppressing function and AJ localization of merlin. The importance of merlin function in AJ establishment was confirmed in primary epithelial keratinocytes, a prototypical system for the study of AJs. Importantly, loss of contact-dependent inhibition of growth and lack 
of AJs appear to be signatures of Nf2 deficiency as these properties are also exhibited by primary $\mathrm{Nf2}^{-/-}$mouse osteoblasts, which are target cells for tumor development in $\mathrm{Nf}^{+/-}$mice (A.I. McClatchey, D. Lallemand, and $M$. Loeffler, unpubl.).

Our data suggest a model whereby merlin stabilizes the link between the AJ and the actin cytoskeleton. It has been demonstrated that prior to AJ assembly, core AJ complexes, many of which are already tethered to the actin cytoskeleton, are diffusely localized throughout the plasma membrane (Adams et al. 1998; Sako et al. 1998). Upon heterotypic interaction, cadherin complexes cluster via lateral movement, concomitant with strengthened cytoskeletal interaction. The presence of apparently normal levels of diffusely localized core AJ complexes in the membranes of $\mathrm{Nf2}^{-/-}$cells suggests that merlin is required for this late step in assembling or stabilizing the final AJ structure.

The establishment and maturation of AJs has been best studied in epithelial cells, where it is clear that this is a complex and dynamic process that begins with the initial establishment of contact between adjacent membranes. A nascent AJ then serves as a nucleus from which the development of new AJs progresses outward, sealing the two membranes (Vasioukhin et al. 2000; Ehrlich et al. 2002). The actin cytoskeleton is required for this process (Angres et al. 1996; Vaezi et al. 2002). In epithelial cells, interdependency between the cortical actin ring and $\mathrm{AJ}$ establishment has been demonstrated (Adams et al. 1998; Quinlan and Hyatt 1999). We show here that merlin localizes to AJs and associates with $\mathrm{AJ}$ components. Merlin has also been reported to physically interact with and stabilize actin filaments in vitro (James et al. 2001). Thus, merlin may function to locally stabilize actin filaments at the site of AJs. Consistent with this idea, we found that loss of merlin in primary keratinocytes led to disruption of the cortical actin ring and disruption of AJs.

We have recently demonstrated a reciprocal relationship between merlin and Rac, a member of the Rho family of GTPases, key regulators of the actin cytoskeleton (for review of Rho GTPases, see Hall 1998; Shaw et al. 2001). Thus, Rac activation leads to phosphorylation and inactivation of merlin; conversely, active merlin can negatively regulate Rac-induced signaling and cell transformation (Shaw et al. 2001). Several studies establish a dynamic relationship between Rac activity and AJ assembly and stabilization (Fukata and Kaibuchi 2001; Lambert et al. 2002; Yap and Kovacs 2003). It has recently been demonstrated that Rac is physically recruited to sites of nascent $\mathrm{AJ}$ assembly and then is removed quickly as maturation proceeds (Ehrlich et al. 2002). In contrast, both constitutively active (S518A) and inactive (S518D) forms of merlin can localize to AJs (D. Lallemand and A. McClatchey, unpubl.). Therefore, Rac may be transiently recruited to nascent AJs to locally inhibit merlin function and facilitate actin remodeling at developing AJs. Subsequent loss of Rac-mediated inhibition of merlin, together with reactivation, perhaps via the activity of a phosphatase, would stabilize the final AJ structure. Active AJ-associated merlin would then maintain low local levels of active Rac. This is consistent with our observation that the levels of active hypophosphorylated merlin are markedly increased in confluent fibroblasts (Shaw et al. 1998). This model predicts that in the absence of merlin, AJs would not be stabilized.

Consistent with the idea that merlin stabilizes the interaction between the AJ and actin cytoskeleton, there are striking similarities between merlin and $\alpha$-catenin. Deletion of $\alpha$-catenin in murine keratinocytes leads to defective AJ formation, loss of contact-dependent inhibition of proliferation, and the development of tumorlike lesions in vivo (Vasioukhin et al. 2001). In fact, as in $\mathrm{Nf2}^{-/-}$cells, AJ components do exhibit membrane localization in $\alpha$-catenin-null keratinocytes; like $\mathrm{Nf2}^{-/-}$cells, $\alpha$-catenin-null cells also exhibit altered actin organization (Vasioukhin et al. 2001). Merlin, like $\alpha$-catenin, may be a key element linking cadherin-containing complexes to the actin cytoskeleton. Actin-controlled assembly of macromolecular complexes at the cell periphery represents a novel mechanism of tumor-suppressor function.

It has recently been proposed that metastatic potential may be conferred by the nature of early or initiating tumor mutations rather than by secondary mutation of metastasis-specific genes (Bernards and Weinberg 2002). $N f 2$ is a clear example of a gene that, when mutated, can contribute to both tumor initiation and progression, depending on the tumor type. Schwannomas are generally slow-growing, benign tumors that rarely metastasize, regardless of their genotype. In contrast, $\mathrm{Nf2}^{+/-}$mice develop a variety of tumors that are highly metastatic (McClatchey et al. 1998). Loss of AJ function has been firmly linked to both tumor development and tumor invasion in many systems (for review, see Cavallaro and Christofori 2001). Finally, the ability of $\mathrm{Nf2}^{-/-}$cells to grow slowly in the absence of added growth factors may provide a unique advantage for successful late-stage metastatic colonization. We suggest that the unique cocktail of growth advantages exhibited by NF2-deficient cells explains the tumorigenic and often metastatic consequences of its loss. Merlin function is critical in many cell types in the mouse, suggesting that merlin inactivation by genetic or other means may be a key step in the development of many types of cancer.

\section{Materials and methods}

\section{Cell culture}

We examined the consequences of both constitutional and acute deletion of Nf2 in MEFs by harvesting primary MEF populations from either embryonic day 12.5 (E12.5) chimeric $N f 2^{-/-:+/+}$embryos or $N f 2^{\text {flox } 2 / f l o x 2}$ embryos. Selection of $N f 2^{-/-}$ MEFs from chimeric embryos has been described (Shaw et al. 2001). Wild-type MEF populations selected from chimeric embryos partially composed of neomycin-resistant wild-type cells were used as controls. Alternatively, $N f 2^{f l o x 2 / f l o x 2}$ MEFs were infected with an adenovirus expressing the Cre recombinase (Ad5CMV-Cre). Mock-infected $N f 2^{\text {flox2/flox2 }}$ MEFs were processed in parallel. Greater than $90 \%$ recombination efficiency was achieved as assessed by Western blot and PCR analysis 
(data not shown). Wild-type (un-Cre-able) MEFs infected with Ad-Cre did not exhibit a detectable phenotype in our assays (data not shown). After establishing that $\mathrm{Nf}^{-/-}$MEFs isolated by either method exhibited identical growth properties, we used Ad-Cre and mock-infected $N f^{\text {flox2/flox2 }} \mathrm{MEFs}$ for subsequent experiments. Primary MEFs were used between passages 2 and 5. $\mathrm{cd} 44^{-/-}$and $\mathrm{Nf1^{-/- }}$ MEFs were isolated from E12.5 $\mathrm{cd} 44^{-{ }^{--}}$or $\mathrm{Nf1} 1^{-1-}$ embryos.

The protocol for mouse keratinocyte preparation was kindly provided by Stuart Yuspa (NCI) and modified slightly. Briefly, after sacrifice, newborn (P1-P2) mouse skin was removed and placed into calcium-free trypsin overnight $\left(4^{\circ} \mathrm{C}\right)$. The following day, the epidermis was separated from the dermis and disaggregated in keratinocyte media (GIBCO-BRL) containing $1.4 \mathrm{mM}$ $\mathrm{CaCl}_{2}$. After a brief centrifugation, the cells were resuspended in keratinocyte media containing $4 \mathrm{ng} / \mathrm{mL}$ EGF, $25 \mu \mathrm{g}$ of bovine pituitary extract, and $0.2 \mathrm{mM} \mathrm{CaCl}_{2}$ and seeded onto coverslips. The following day, the concentration of calcium in the media was lowered to $0.05 \mathrm{mM}$. For AJ induction, calcium was raised to $2 \mathrm{mM}$ for $20 \mathrm{~h}$. Ad-Cre infection of keratinocytes was performed as for MEFs.

\section{Adenovirus production and infection}

Ad5CMV-Cre was purchased from University of Iowa Gene Transfer Vector Core. The Blue Box $\left(N f 2^{\Delta B B}\right), N f 2^{S 518 A}$, and $N f 2^{L 64 P}$ mutants were created by site-directed mutagenesis of mouse Nf2 isoform 1 (QuikChange, Stratagene) in pcDNA3. All constructs were sequenced in their entirety. The $N f 2^{\Delta B B}$-expressing adenovirus was prepared using the Adeasy system (Stratagene) according to the manufacturer. Infections were performed overnight at a concentration of 50 m.o.i./cell.

\section{GFP-fusion constructs}

Wild-type and L64P-containing mouse Nf2 cDNAs were cloned in-frame into pEGFP-C1 expression vectors to create $\mathrm{N}$-terminal GFP-fusions (Clontech). Keratinocytes were transfected using Lipofectamine Plus (GIBCO-BRL).

\section{Proliferation analysis}

For cell growth curves, $4 \times 10^{4} \mathrm{MEFs}$ were seeded in triplicate in 15-mm wells in DME $+10 \%$ FBS. Beginning the following day (day 1), cells were trypsinized and counted daily. Experiments were repeated at least three times using multiple preparations of MEFs. For FACs analysis, MEFs were either maintained in DME $+10 \%$ FBS for $48 \mathrm{~h}$ after reaching confluence, suspended in DME $+10 \%$ FBS for $16 \mathrm{~h}$, or maintained in DME $+10 \%$ FBS for $24 \mathrm{~h}$ after $120 \mathrm{~J}$ of UVC, labeled with $\operatorname{BrdU}(10 \mathrm{mM}, 4 \mathrm{~h})$, and collected and processed according to the manufacturer (BectonDickinson). Briefly, cells were fixed in $70 \%$ ethanol and treated with $2 \mathrm{~N} \mathrm{HCl}$ in $0.5 \%$ Triton X-100. After neutralization with $0.1 \mathrm{M} \mathrm{Na}_{2} \mathrm{~B}_{4} \mathrm{O}_{7}(\mathrm{pH} 8.5)$, MEFs were sequentially incubated with an anti-BrdU antibody (Becton-Dickinson; $30 \mathrm{~min}$ ) and FITC-conjugated horse anti-mouse secondary antibody (Vector Laboratories; $30 \mathrm{~min}$ ). Cells were resuspended in PBS containing $40 \mu \mathrm{g} / \mathrm{mL}$ propidium iodide and RNAse. Analysis was performed with CellQuest software (Becton-Dickinson).

\section{Protein analysis}

Total protein extracts were prepared in modified RIPA lysis buffer (50 mM Tris at pH 7.4, 1\% Triton X-100, 0.1\% SDS, 0.5\% sodium deoxycholate, $150 \mathrm{mM} \mathrm{NaCl}, 1 \mathrm{mM} \mathrm{Na}_{3} \mathrm{VO}_{4}, 10 \mathrm{mM}$ $\mathrm{NaF}, 10 \mathrm{mM} \beta$-glycerophosphate, $1 \mathrm{mM}$ EDTA at pH 8.0, $1 \mathrm{mM}$
EGTA, and protease inhibitors). For membrane extracts, cells were lysed by mechanical disruption in cold hypotonic buffer (10 mM HEPES at pH 7.4, 1 mM EDTA, and protease inhibitors). Nuclei were pelleted by centrifugation at $750 \mathrm{~g}$ for $10 \mathrm{~min}$. Further centrifugation of the resulting supernatant at $1 \times 10^{5} \mathrm{~g}$ for 1 $\mathrm{h}$ led to recovery of the cytosolic fraction $(\mathrm{C})$. The pellet was extracted with membrane extraction buffer $(\mathrm{MEB} ; 50 \mathrm{mM}$ Tris at $\mathrm{pH} 7.4,1 \%$ Triton $\mathrm{X}-100,150 \mathrm{mM} \mathrm{NaCl}, 1 \mathrm{mM}$ EDTA, $1 \mathrm{mM}$ $\mathrm{Na}_{3} \mathrm{VO}_{4}$, and protease inhibitors) and centrifuged at $1 \times 10^{5} \mathrm{~g}$ for $1 \mathrm{~h}$. This supernatant corresponded to the Triton X-100 soluble membrane extract (S). The final pellet was extracted with modified RIPA buffer and centrifuged at $1 \times 10^{5} \mathrm{~g}$ for $5 \mathrm{~min}$; this supernatant corresponded to the Triton X-100 insoluble fraction (I). Equal quantities of protein were separated by SDS-PAGE, transferred to nitrocellulose membrane, and probed at $4{ }^{\circ} \mathrm{C}$ overnight in PBS, $0.05 \%$ Tween-20, and $5 \%$ nonfat dry milk or $1 \%$ BSA for phospho-specific antibodies.

\section{Antibodies}

The primary antibodies used were from Santa Cruz (anti-merlin: sc331, sc332, 1:500 dilution; anti-JNK1: sc474, 1:1000), Cell Signaling (anti-ERK1/2: 9102; anti-phosphoERK1/2: 9101; antiphospho-JNK: 9255, all at a 1:500 dilution), Sigma (anti-tubulin: T9026), Zymed (anti-E-cadherin: 13-1900), and Transduction Labs (anti- $\beta$-catenin: C19220; anti- $\alpha$-catenin: C21620; anti-Ncadherin: C70320; anti-paxillin: P13520; anti-P120: P17920). Antibodies to c-jun and cyclin D1 were gifts from M. Yaniv (Institute Pasteur, Paris, France) and E. Harlow (Harvard Medical School, Boston, MA), respectively. HRP-linked secondary antibodies to rabbit, mouse, or rat were from Amersham.

\section{Indirect immunofluorescence}

Confluent, serum-starved wild-type MEFs were simultaneously permeabilized and fixed as follows: Cells plated on coverslips were incubated in $1 \%$ paraformaldehyde, $0.1 \%$ Triton X-100, $0.1 \% \mathrm{NP}-40$ in $2.5 \mathrm{mM}$ triethanolamine for $30 \mathrm{~min}$. Cells were then further permeabilized in $0.5 \%$ Triton X-100 in PBS for 10 min. Coverslips were blocked in PBS $+0.2 \%$ BSA and incubated with anti- $\beta$-catenin (1:200), anti-merlin (sc332; $1: 50)$, or anti-Ecadherin $(1: 250)$ primary antibodies at $4^{\circ} \mathrm{C}$ overnight in PBS $+0.2 \%$ BSA. Coverslips were rinsed five times with PBS and incubated with secondary antibodies in PBS $+0.2 \%$ BSA. FITC- and rhodamine-conjugated secondary anti-mouse, antirabbit, and anit-rat antibodies were purchased from Calbiochem or ICN (anti-rat). Coverslips were mounted using Vectashield (Vector Labs). Photographs were taken at 20x with a Nikon Microphot epifluorescence microscope.

\section{Immunoprecipitation}

For immunoprecipitation, $200 \mu \mathrm{g}$ of Triton X-100 soluble membrane extract was precleared at $4^{\circ} \mathrm{C}$ for $1 \mathrm{~h}$ with $20 \mu \mathrm{L}$ of protein-G Sepharose (Pharmacia) and $2 \mu \mathrm{g}$ of either mouse or rabbit nonspecific IgG (Sigma) in a final volume of $500 \mu \mathrm{L}$ of MEB. After quick centrifugation, the supernatant was incubated at $4^{\circ} \mathrm{C}$ for $2 \mathrm{~h}$ with $20 \mu \mathrm{L}$ of protein-G Sepharose and $2 \mu \mathrm{g}$ of anti-merlin (sc331, sc332), anti- $\beta$-catenin, anti- $\alpha$-catenin, P120, anti-E-cadherin, or anti-N-cadherin antibodies. The beads were washed five times in cold MEB and resuspended in $50 \mu \mathrm{L}$ of $1 \times$ Laemmli SDS sample buffer.

\section{Acknowledgments}

We thank Nick Dyson, Spyros Artavanis-Tsakonas, and Jeff Settleman for critical reading of the manuscript, Jim Rocco and 
members of his laboratory for help with adenovirus preparation, Ivan Stamenkovic for the $c d 44^{-/-}$mice, and Brett Johnson and Raphaella Sordella for useful discussions and reagents. We thank Stuart Yuspa and Ulriche Lichti for helpful advice in isolating and culturing primary murine keratinocytes. We gratefully acknowledge members of the McClatchey laboratory for helpful discussions throughout. This study was supported by the American Cancer Society, the Department of Defense Neurofibromatosis Program (A.I.M.), Ligue Contre le Cancer (M.G.), and the National Neurofibromatosis Foundation and Association pour la Recherche Contre le Cancer (D.L.).

The publication costs of this article were defrayed in part by payment of page charges. This article must therefore be hereby marked "advertisement" in accordance with 18 USC section 1734 solely to indicate this fact.

\section{References}

Adams, C.L., Chen, Y.T., Smith, S.J., and Nelson, W.J. 1998. Mechanisms of epithelial cell:cell adhesion and cell compaction revealed by high-resolution tracking of E-cadheringreen fluorescent protein. J. Cell Biol. 142: 1105-1119.

Angres, B., Barth, A., and Nelson, W.J. 1996. Mechanism for transition from initial to stable cell-cell adhesion: Kinetic analysis of E-cadherin-mediated adhesion using a quantitative adhesion assay. J. Cell Biol. 134: 549-557.

Bernards, R. and Weinberg, R.A. 2002. A progression puzzle. Nature 418: 823.

Bretscher, A., Edwards, K., and Fehon, R.G. 2002. ERM proteins and merlin: Integrators at the cell cortex. Nat. Rev. Mol. Cell Biol. 3: 586-599.

Cavallaro, U. and Christofori, G. 2001. Cell adhesion in tumor invasion andmetastasis: Loss of the glue is not enough. Biochim. Biophys. Acta 1552: 39-45.

Cichowski, K. and Jacks, T. 2001. NF1 tumor suppressor gene function: Narrowing the GAP. Cell 104: 593-604.

Cichowski, K., Santiago, S., Jardim, M., Johnson, B.W., and Jacks, T. 2003. Dynamic regulation of the Ras pathway via proteolysis of the NF1 tumor suppressor. Genes \& Dev. 17: 449-454.

Ehrlich, J.S., Hansen, M.D.H., and Nelson, J.W. 2002. Spatiotemporal regulation of Rac1 localization and lamellipodial dynamics during epithelial cell:cell adhesion. Dev. Cell 3: $259-270$.

Fernandez-Valle, C., Tang, Y., Ricard, J., Rodenas-Ruano A., Taylor, A., Hackler, E., Biggerstaff, J., and Iacovelli, J. 2002. Paxillin binds schwannomin and regulates its density-dependent localization and effect on cell morphology. Nat. Genet. 3: 354-362.

Fukata, M. and Kaibuchi, K. 2001. Rho-family GTPases in cadherin-mediated cell-cell adhesion. Nat. Rev. Mol. Cell Biol. 2: 887-896.

Giovannini, M., Robanus-Maandag, E., van der Valk, M., NiwaKawakita, M., Abramowski, V., Goutebroze, L., Woodruff, J.M., Berns, A., and Thomas, G. 2000. Conditional biallelic Nf2 mutation in the mouse promotes manifestations of human neurofibromatosis type 2. Genes \& Dev. 14: 1617-1630.

Gloushankova, N.A., Krendel, M.F., Alieva, N.O., Bonder, E.M., Feder, H.H., Vasiliev, J.M., and Gelfand, I.M. 1998. Dynamics of contacts between lamellae of fibroblasts: Essential role of the actin cytoskeleton. Proc. Natl. Acad. Sci. 95: 43624367.

Gutmann, D.H. 1997. Molecular insights into neurofibromatosis 2. Neurobiol. Dis. 3: 247-261.

Gutmann, D.H., Geist, R.T., Xu, H.-M., Kim, J.S., and Saporito-
Irwin, S. 1998. Defects in Neurofibromatosis 2 protein function can arise at multiple levels. Hum. Mol. Genet. 7: 335-345.

Gutmann, D.H., Sherman, L., Seftor, L., Haipek, C., Hoang, L.K., and Hendrix, M. 1999. Increased expression of the NF2 tumor suppressor gene product, merlin, impairs cell motility, adhesion and spreading. Hum. Mol. Genet. 8: 267-275.

Hall, A. 1998. Rho GTPases and the actin cytoskeleton. Science 279: 509-514.

James, M.F., Manchanda, N., Gonzalez-Agosti, C., Hartwig, J.H., and Ramesh, V. 2001. The Neurofibromatosis 2 protein product merlin selectively binds F-actin but not G-actin, and stabilizes the filaments through a lateral association. Biochem. J. 356 (Pt2): 377-386.

Johnson, K.C., Kissil, J.L., Fry, J.L., and Jacks, T. 2002. Cellular transformation by a FERM domain mutant of the Nf2-tumor suppressor gene. Oncogene 21: 5990-5997.

Kalamarides, M., Niwa-Kawakita, M., Leblois, H., Abramowski, V., Perricaudet, M., Janin, A., Thomas, G., Gutmann, D.H., and Giovannini, M. 2002. Nf2 gene inactivation in arachnoidal cells is rate-limiting for meningioma development in the mouse. Genes \& Dev. 16: 1060-1065.

LaJeunesse, D.R., McCartney, B.M., and Fehon, R.G. 1996. Structural analysis of Drosophila merlin reveals functional domains important for growth control and subcellular localization. J. Cell Biol. 141: 1589-1599.

Lambert, M., Choquet, D., and Mege, R.-M. 2002. Dynamics of ligand-induced, Rac1-dependent anchoring of cadherins to the actin cytoskeleton. J. Cell Biol. 157: 469-479.

Maeda, M., Matsuim, T., Imamuram, M., Tsukitam, S., and Tsukita, S. 1999. Expression level, subcellular distribution and $\rho$-GDI binding affinity of merlin in comparison with ezrin/radixin/moesin proteins. Oncogene 18: 4788-4797.

McCartney, B.M. and Fehon, R.G. 1996. Distinct cellular and subcellular patterns of expression imply distinct functions for the Drosophila homologues of moesin and the neurofibromatosis 2 tumor suppressor, merlin. I. Cell Biol. 133: 843-852.

McClatchey, A.I., Saotome, I., Ramesh, V., Gusella, J.F., and Jacks, T. 1997. The Nf2 tumor suppressor gene product is essential for extraembryonic development immediately prior to gastrulation. Genes \& Dev. 11: 1253-1265.

McClatchey, A.I., Saotome, I., Mercer, K., Crowley, D., Gusella, J.F., Bronson, R.T., and Jacks, T. 1998. Mice heterozygous for a mutation at the Nf2 tumor suppressor locus develop a range of highly metastatic tumors. Genes \& Dev. 12: 1121 1133.

Morrison, H., Sherman, L.S., Legg, J., Banine, F., Isacke, C., Haipek, C.A., Gutmann, D.H., Ponta, H., and Herrlich, P. 2001. The NF2 tumor suppressor gene product, merlin, mediates contact inhibition of growth through interactions with CD44. Genes \& Dev. 15: 968-980.

Nagafuchi, A. 2001. Molecular architecture of AJs. Curr. Opin. Cell Biol. 13: 600-603.

Nollet, F., Berx, G., and van Roy, F. 2000. The role of the Ecadherin/catenin adhesion complex in the development and progression of cancer. Mol. Cell. Biol. Res. Comm. 2: 77-85.

Obremski, V.J., Hall, A.M., and Fernandez-Valle, C. 1998. Merlin, the neurofibromatosis type 2 gene product, and $\beta 1$ integrin associate in isolated and differentiating Schwann cells. J. Neurobiol. 37: 487-501.

Pelton, P.D., Sherman, L.S., Rizvi, T.A., Marchionni, M.A., Wood, P., Friedman, R.A., and Ratner, N. 1998. Ruffling membrane, stress fiber, cell spreading and proliferation abnormalities in human Schwannoma cells. Oncogene 17: 2195-2209.

Perez-Moreno, M., Jamora, C., and Fuchs, E. 2003. Sticky busi- 
Lallemand et al.

ness. Orchestrating cellular signals at adherens junctions. Cell 112: 535-548.

Quinlan, M.P. and Hyatt, J.L. 1999. Establishment of the circumferential actin filament network is a prerequisite for localization of the cadherin-catenin complex in epithelial cells. Cell Growth Diff. 10: 839-854.

Sako, Y., Nagafuchi, A., Tsukita, S., Takeichi, M., and Kasumi, A. 1998. Cytoplasmic regulation of the movement of E-cadherin on the free cell surface as studied by optical tweezers and single particle tracking: Corralling and tethering by the membrane skeleton. J. Cell Biol. 140: 1227-1240.

Shaw, R.J, McClatchey, A.I., and Jacks, T. 1998. Regulation of the neurofibromatosis type 2 tumor suppressor protein, merlin, by adhesion and growth arrest stimuli. J. Biol. Chem. 273: 7757-7764.

Shaw, R.J. Paez, J.G., Curto, M., Yaktine, A., Pruitt, W.M., Saotome, I., O'Bryan, J.P., Gupta, V., Ratner, N., Der, C.J., et al. 2001. The Nf2 tumor suppressor, merlin, functions in Racdependent signaling. Dev. Cell 1: 63-72.

Sun, C.-X., Robb, V.A., and Gutmann, D.H. 2002. Protein tumor suppressors: Getting a FERM grip on growth regulation. J. Cell Sci. 115: 3991-4000.

Vaezi, A., Bauer, C., Vasioukhin, V., and Fuchs, E. 2002. Actin cable dynamics and Rho/Rock orchestrate a polarized cytoskeletal architecture in the early steps of assembling a stratified epithelium. Dev. Cell 3: 367-381.

Vasioukhin, V., Bauer, C., Yin, M., and Fuchs, E. 2000. Directed actin polymerization is the driving force for epithelial cellcell adhesion. Cell 100: 209-219.

Vasioukhin, V., Bauer, C., Degenstein, L., Wise, B., and Fuchs, E. 2001. Hyperproliferation and defects in epithelial polarity upon conditional ablation of $\alpha$-catenin in skin. Cell 104: 605-617.

Yap, A.S. and Kovacs, E.M. 2003. Direct cadherin-activated cell signaling: A view from the plasma membrane. J. Cell Biol. 160: $11-16$.

Yonemura, S., Itoh, M., Nagafuchi, A., and Tsukita, S. 1995. Cell-to-cell adherens junction formation and actin filament organization: Similarities and differences between non-polarized fibroblasts and polarized epithelial cells. J. Cell Sci. 108: $127-142$. 


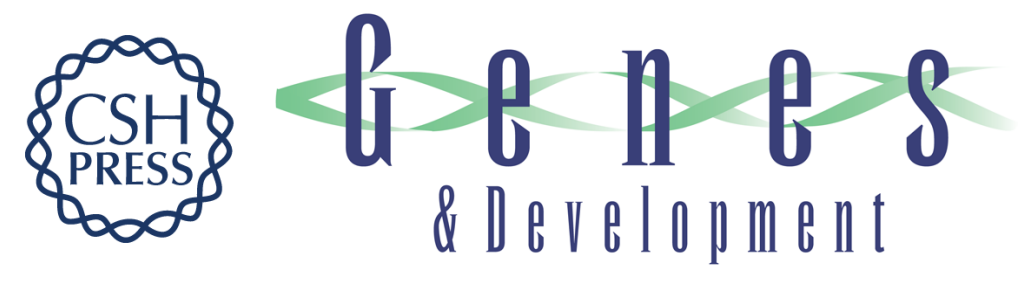

\section{NF2 deficiency promotes tumorigenesis and metastasis by destabilizing adherens junctions}

Dominique Lallemand, Marcello Curto, Ichiko Saotome, et al.

Genes Dev. 2003, 17:

Access the most recent version at doi:10.1101/gad.1054603

References This article cites 39 articles, 18 of which can be accessed free at: http://genesdev.cshlp.org/content/17/9/1090.full.html\#ref-list-1

License

Email Alerting

Receive free email alerts when new articles cite this article - sign up in the box at the top Service right corner of the article or click here.

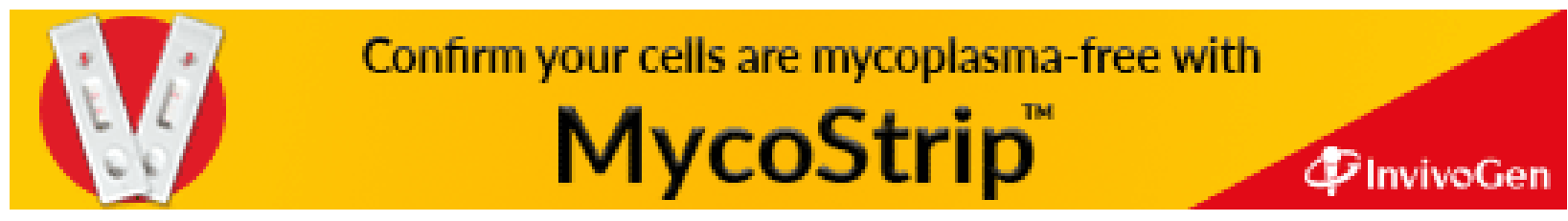

